\title{
Implementation of Human Face Detection System for Door Security using Raspberry Pi
}

\author{
Shrutika V. Deshmukh ${ }^{1}$, Prof Dr. U. A. Kshirsagar ${ }^{2}$ \\ P.G Student, Electronics and Telecommunication Dept, HVPM's College of Engineering and Technology
}

Amravati, India ${ }^{1}$

Professor and HOD, Electronics and Telecommunication Department, HVPM's College of Engineering and

Technology, Amravati, India ${ }^{2}$

\begin{abstract}
Nowadays the number of thefts and identity fraud has become a serious issue. In order to avoid these thefts and identity fraud, a face recognition system must be established. The scope of this project is to develop a security access control application based on face recognition. The haar-like features is used for face detection and HOG +SVM algorithm is used for face recognition. In order to achieve a higher accuracy and effectiveness we use OpenCV libraries and python computer language. Training and identification is done in embedded device known as Raspberry Pi.
\end{abstract}

Keywords: Face detection, Face recognition, raspberry pi, security.

\section{INTRODUCTION}

In this current time a lot of incident occurs like robbery, stealing unwanted entrance happens abruptly. So the security does matters in this daily life. People always remain busy in their day to day work also wants to ensure their safety of their beloved things. Sometimes they forget to look after their necessary things like keys, wallet, credit cards etc. Without these, they are unable to access their home or any place they want.Traditional security system require the user a key, a security password, an RFID card, or ID card to have access to the system. However, these security systems have deficiencies; for example, they can be forgotten or stolen from unauthorized people. As a result, there is a need to develop software that guarantees a higher security level is a template. One of the unique features of our brain is that it can think only in images not in words. Once you may forget to keep your Car's key but you will never forget to bring a face with you.God has given everyone a unique face. Face is the most important part of our body, so that it can reflect many emotions of a person. From a long year ago, we are using non-living thing (smart cards, plastic cards, PINS, tokens, keys) for authentication and to get grant access in restricted areas like ISRO, NASA, and DRDO etc. There are two types of biometric as physiological characteristics (face, fingerprint, finger geometry, hand geometry, palm, iris, ear and voice) and behavioural characteristics (gait, signature and keystroke dynamics). Sometimes your behavioural traits may changes because of illness, fear, hunger etc. Face detection and recognition system is more cheap, simple, accurate and non-intrusive process as compare to other biometrics. The system will fall into two categories as face detection (1:1) and face recognition(1:N).In the face detection we have to classify between face versus non face region while in recognition process we have to compare that single face image with multiple images from the input image. In This work uses BCM2835 processor, popularly known as Raspberry pi Board. The core of the board is the above processor. It is a RISC processor based on ARM11. The board has special features like camera interface and touch screen that make it suitable for real time image processing Open cv consists of huge number of inbuilt functions for image processing. It is under BSD license and hence libraries are free of proprietary cost. The full-fledged library functions simplify the complex mathematical operations.

\section{LITERATURE REVIEW}

Since there are number of works have been done using raspberry pi models in digital image processing field. Like image capturing technique in an embedded system with Raspberry Pi 1 Model B. Especially the biometric access systems like voice based access, speaker recognition, password key systems, standalone face recognition system etc. all using Raspberry pi 1 model B or B+. Moreover the face recognition system are worked deeply for the security purpose and surveillance and calculation of different parameters like false rejection rate and false acceptance rate are done as an aspect as non-living things such as smart-cards, plastic cards, PINS, tokens, keys are used for authentication. Hence the performance, random speed, etc. other parameters are based with respect to the hardware design of Raspberry pi 1 Model B and Raspberry Pi model B+. Also Raspberry pi is known for its versatility and inexpensiveness with respect to 


\section{ISO 3297:2007 Certified}

Vol. 5, Issue 4, April 2017

display modules. As time passes public utilizes the Raspberry Pi 1 Model B+ as the central module and extract the results.

[1] K.Gopalakrishnan, V. Sathish Kumar : They were usedan Embedded platform which was very unique and easy to implement .They proposed an image capturing technique in an embedded system based on Raspberry Pi board and Considering the requirements of image capturing and recognition algorithm, Raspberry Pi processing module and its peripherals, implementing based on this platform, Finally they were concluded that the designed system is fast enough to run the image capturing, recognition algorithm, and the data stream can flow smoothly between the camera and the Raspberry Pi board.

[2] Tony Di Cola: His project was "Raspberry Pi Face Recognition in Treasure Box" a great example of how to use the Raspberry Pi and Pi camera with Open CV's computer vision algorithms. By compiling the latest version of Open $\mathrm{CV}$, it can get access to the latest and most interesting computer vision algorithms like face recognition. Also he used a Solenoid double action lock which is lock/unlock using key after power off.

[3] KuldeepSoni:Hedeveloped a system withan advanced surveillancecamera capable of face detection and atthe same time recognizing the face detected using OPEN-CV library, Eigen face methodology and these all processing has been done on RaspbianOS on Raspberry Pi. For capturing the images he used Pi Camera Board. With the help offace recognition capability he proved that the advanced surveillance camera system using face detection and atthe same time recognizing the face detected is highly secured system.

[4] MedakTeenaRavali, Prof. RangaSaiKomaragiri: They proposed that a low cost alternative for DSP kits for image processing using Raspberry pi board with Open-cv package. In this proposed work the platform for image processing is or algorithm for face recognition is implemented on principal component analysis. There system has been designed on the criteria of resources optimization, low power consumption and improved operation speed.

[5] Anoop Mishra, Arshita Dixit: They proposed there work on Raspberry pi 2 B+ model with camera interface to capture an image and convert this capture image into gray image with digital processing image algorithm. Also they conclude that the practically applied result is rational and designed system is technically smarter than while performing the image interfacing system on a Personal Computer.

[6] KandlaArora: He proposed that real time application of Face Recognition concept by generating a matlab code using image acquisition tool box on the basic approach used is PCA using Eigen faces.

[7] Sanjana Prasad, P. Mahalakshmi, A. John Clement Sunder, R. Swathi:They implement Smart surveillance monitoring system using Raspberry pi and PIR sensor for mobile devices.

Also their proposed work implements home security system captures information and transmits it via a $3 \mathrm{G}$ Dongle to a Smart phone using web application. Raspberry pi operates and controls motion detectors and video cameras for remote sensing and surveillance, streams live video and recordsit for future playback.

[8]AjinkyaPatil, MrudangShukla:they were used their face recognition system for student attendance in their class for avoid wastage of time by using automatic process which is based on image processing and face detection \&face recognition system. This face detection differentiates faces from non-faces and is therefore essential for accurate attendance. The other strategy involves face recognition for marking the student's attendance. The student database is collected. The database includes name of the students, there images \&roll number.

[9] Manal Abdullah, MajdaWazzan and SaharBo-saeed: They proposed Principle Component Analysis PCA is a classical feature extraction and data representation technique widely used in pattern recognition. Their paper conducts a study to optimize the time complexity of PCA (Eigen faces) that does not affects the recognition performance applying their proposed enhanced algorithm.

[10]Raspberry Official site - http://www.raspberrypi.org

\section{HARDWARE USED}

\section{RASPBERRY PI:}

To implement such a project, the main and most important step was finding the hardware to use for the device. We have chosen a Raspberry Pi model B3 to use in our device. We have done a lot of research, and compared elements in different microcontrollers, like, cost, processing, and user friendliness. The main reasons why we have chosen this specific element are the high processing capacity, relatively low price, and its ability to adapt in different programming modes. The device uses Linux as an operating system, which has access to a large number of libraries and applications compatible with it. Raspberry Pi has an Ethernet port allowing us a network connection, as long as we are in the same subnet with the device we want to access and manage, 4 USB ports used to connect devices like a keyboard, mouse, camera, and other devices that connect through a USB port, and an HDMI port giving us access to the interface of the operating system installed, and can also be used the first time while installing the devices.It has 40 pins that allow us to receive and send signals. They are divided in half into two groups: the $3 \mathrm{~V}$, and the $5 \mathrm{~V}$ group. Therefore, one side of the 
microcontroller gives a voltage of $3 \mathrm{~V}$, and the other $5 \mathrm{~V}$. Besides the 40 voltagepins, it has pinsthat are used to receive signals, which in our case was used to connect the button, that will send the signal for the face identification. Raspberry Pi does not have an operating system previously installed, but that can be downloaded from the Raspberry website, and transferred to an SD card, Figure 1 shows the Raspberry pi model B 3 along with its components

The foundation provides Debian and Arch Linux ARM distributions and also Python as the main programming language, with the support for BBC BASIC, $\mathrm{C}$ and Perl. The system is programmed using Python programming language. We have developed algorithms, for face detection and recognition for security.

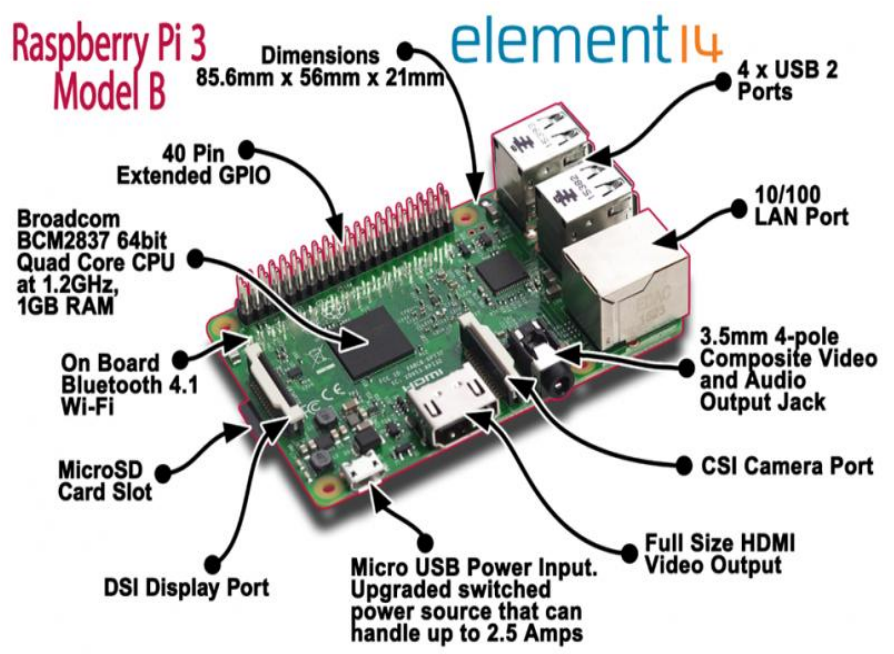

Fig. 1 Raspberry pi model B 3

\section{WORKING}

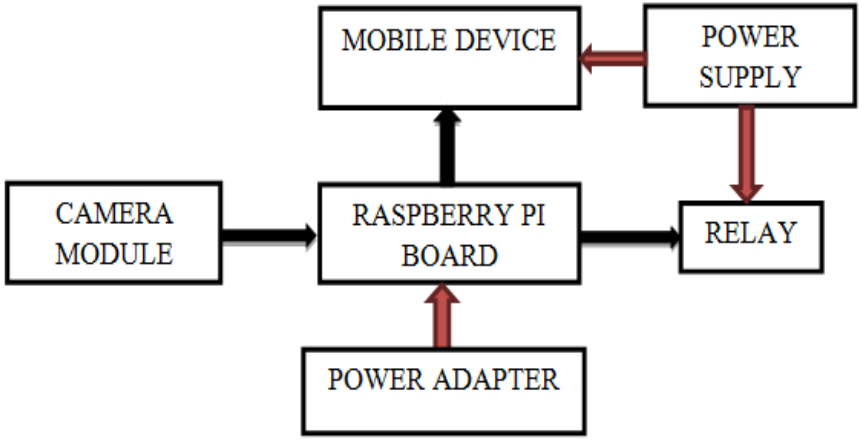

Fig: Block diagram of Implementation of human face detection system for door security using Raspberry Pi

The above fig shows the basic block diagram of the Raspberry pi based face recognition system for door unlocking. Our project system can be operated in two different sections, i.e. one for capturing and creating a data base and the other section is to capture the image and which is used for identifying or comparing theimages in the database. Here in the second section we use hog+ svmalgorithum used face recognition for finding the matches.

Camera module: Camera module is Pi camera interfacing to the raspberry pi module. Its resolution is 5-megapixel and still picture resolution 2592 x 1944, Max image transfer rate 1080p: 30fps, this Pi camera module is used for captures an image and send captured image to the Raspberry pi module. At the first time camera module captures 6 images to create a database of the authorized person as a train faces then secondly take a test face or live captured image to compare with train faces (Data base).

Raspberry pi module: Raspberry pi B3 module is small computer board. When image taken by the raspberry pi it is compared with Eigen face image. At the first time when we capture the image to create a data base raspberry pi module capture six types of the images to create a data base in the system and this data base is compared with the live captured image 
Raspberry pi consist of OPEN CV library in which we can write algorithm in JAVA, Python or C++. After comparing two images output is positive/negative then it gives commands to mobile device.

From power adapter Raspberry pi takes 5V 2A supply.

Mobile Device: It is used to sending a message to the authorities after comparison output is positive or negative. If output is positive then "Person Identified!!DOOR OPENS!!"message send to the authority person otherwise send "UNKNOWN PERSON IS TRYING TO UNLOCK THE DOOR". mobile deviceis connected to the Raspberry pi board via RS 232 to USB convertor. It operates on Single supply voltage $3.4 \mathrm{~V}-4.5 \mathrm{~V}$.

Relay:Here we use a 12V 1A Relay is as an output module which is used to unlock the door when known face is recognizing successfully. When a power is supplied to thecoil, it generates a magnetic force that actuates the switch mechanism.

\section{$>\quad$ Face Detection}

All Face detection is a computer technology being used in a variety of applications that identifies human faces in digital images. Face detection also refers to the psychological process by which humans locate and attend to faces in a visual scene. HAAR Cascade Haar-like features are digital image features used in object recognition. They owe their name to their intuitive similarity with Haar wavelets and were used in the first real-time face detector. Here we will work with face detection. Initially, the the algorithm needs a lot of positive images (images of faces) and negative images (images without faces) to train the classifier. Then we need to extract features from it. For this, haar features shown in below image are used. They are just like our convolutionalkernel. Each feature is a single value obtained by subtracting sum of pixels under white rectangle from sum of pixels under black rectangle. Now all possible sizes and locations of each kernel is used to calculate plenty of features. For each feature calculation, we need to find sum of pixels under white and black rectangles. To solve this, they introduced the integral images. It simplifies calculation of sum of pixels, how large may be the number of pixels, to an operation involving just four pixels

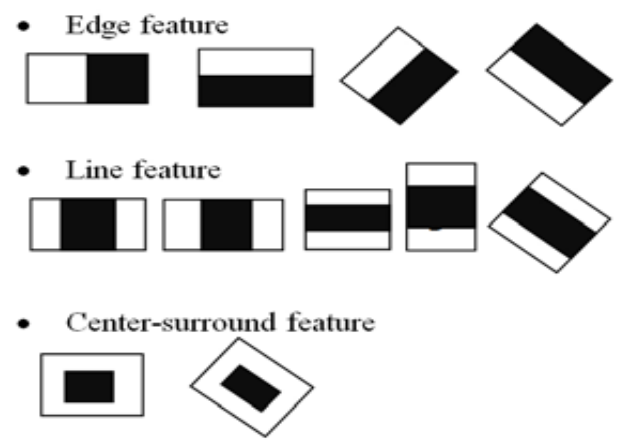

Fig. 2.Haar feature

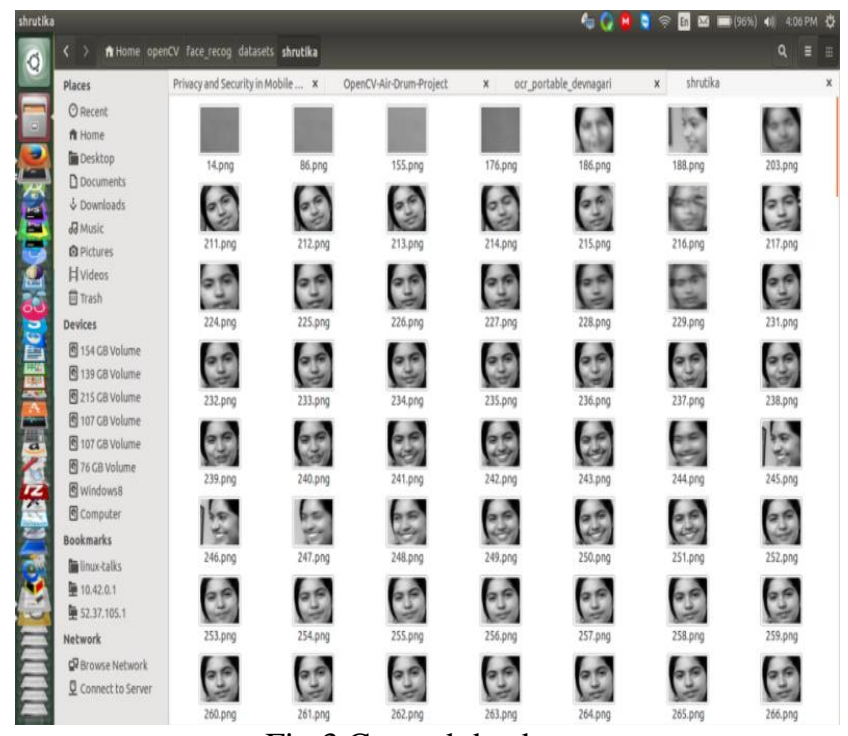

Fig. 3 Created database 
Vol. 5, Issue 4, April 2017

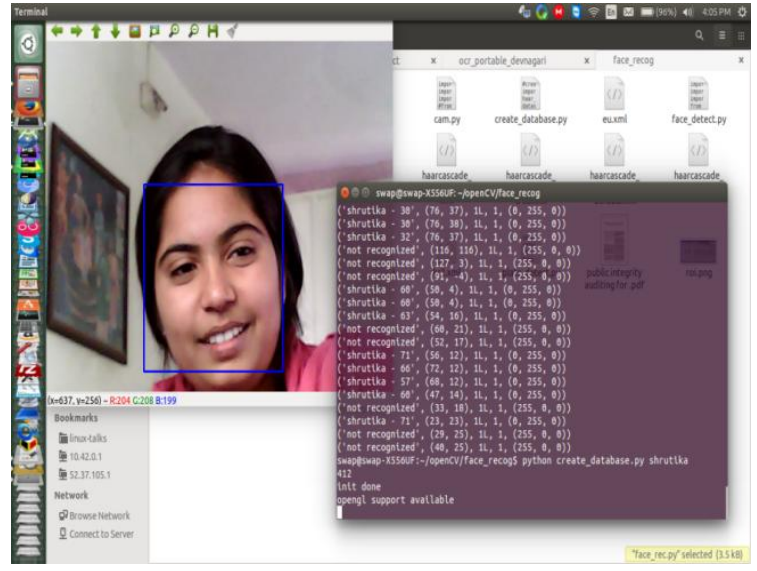

Fig.4.Captured image

\section{$>\quad$ FACE RECOGNIZATION}

The histogram of oriented gradients (HOG) is a feature descriptor usedin computer and image processing for the purpose of recognition The technique counts occurrences of gradient orientation in localized portions of an image. This method is similar to that of edge orientation histograms, scale-invariant feature transform descriptors, and shape contexts, but differs in that it is computed on a dense grid of uniformly spacedcells and uses overlapping local contrast normalization forimproved accuracy.The essential thought behind the histogram of oriented gradients descriptor is that local object appearance and shape within an image can be described by the distribution of intensity gradients or edge directions. The image is divided into small connected regions called cells, and for the pixels within each cell, a histogram of gradient directions is compiled. The descriptor is the concatenation of these histograms. For improved accuracy, the local histograms can be contrast-normalized by calculating a measure of the intensity across a larger region of the image, called a block, and then using this value to normalize all cells within the block. This normalization results in better invariance to changes in illumination and shadowing. The final step in object recognition using histogram of oriented gradient descriptors is to feed the descriptors into some recognition system based on supervised.The support vector machine (SVM) classifier is a binary classifier which looks for an optimal hyper plane as a decision function. Once trained on images containing some particular object, the SVM classifier can make decisions regarding the presence of an object, such as a human, in additional test images. More formally, a support vector machine constructs a hyper plane or set of hyper planes in a high- or infinite-dimensional space, which can be used for classification, regression, or other tasks. Intuitively, a good separation is achieved by the hyper plane that has the largest distance to the nearest training-data point of any class (so-called functional margin), since in general the larger the margin the lower the generalization error of the classifier system shows excellent performance efficiency and can be used for face detection even from poor quality images.

\section{IMPLEMENTATION}

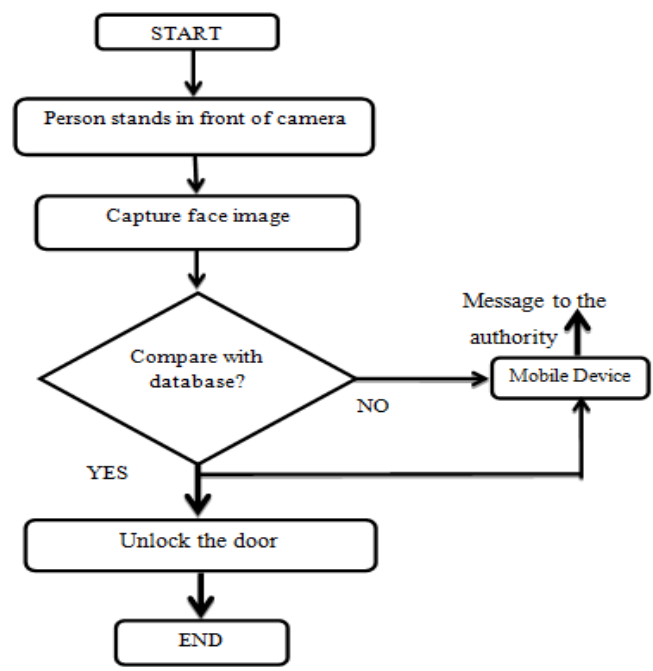

Fig: Flowchart of Implementation of Human Face Detection system for door security using Raspberry Pi 


\section{ISO 3297:2007 Certified}

Vol. 5, Issue 4, April 2017

In above fig 2.3 shows that the flowchart of face captures and recognition process, at initial stage the authorized person comes in front of camera. The camera module will capture the face image with current poses. The captured face of current poses creates a data base ofthe authorized person and stores this. At the next time camera module will capture the current live face of the person. All this process is done in Raspberry pi module.

When comparison done successfully the Relay switch is ON to unlock the door otherwise Raspberry pi module will capture an image once again through the camera and process is repeat Raspberry pi will send a command to the Mobile device send a message to the Authorized person is "FACE MATCHED" when comparison is done successfully otherwise send security alert "Unknown person will try to unlock the door".

\section{CONCLUSION}

The design of the face recognition system using Raspberry pi can make the smaller, lighter and with lower power consumption, so it is more convenient than the PC-based face recognition system. Because of the open source code, it is freer to do software development on Linux. We use

HOG+SVM algorithm for the face recognition and detection process. Also send a security alert message to the authorized person. A face detection system using Raspberry Pi was developed. The system was programmed using Python programming language. Both Real time face detection and face detection from specific images, i.e. object recognition, was carried out. The efficiency of the system was analyzed in terms of face detection rate. The analysis revealed that the present system shows excellent performance efficiency and can be used for face detection even from poor quality images.

\section{FUTURE SCOPE}

Using raspberry pi the current project can be modified by an Infrared camera interfacing it can be used in Smart Surveillance Monitoring security system which any type of public security is using Living body detection or spying, Also it can be used in Attendance system of the class, Also some profound applications can be implemented using interfacing of Raspberry pi and Arduino UNO board like sensor application of smartcard swapping, finger detection, alcohol detection, agriculture humidity sensing, Temperature sensing using web server, and many more. New studies are being made to allow images to be processed on the GPU of the Raspberry Pi, achieving better results with the use of specific libraries.

\section{ACKNOWLEDGMENT}

Thank to Prof Dr. U. A. Kshirsagar, HOD, Electronics and Telecommunication Department, HVPM's College of Engineering and Technology, Amravati, India.

\section{REFERENCES}

[1] S. Prabhakar, S. Pankanti, and A. K. Jain, "Biometric recognition: Security and privacy concerns," IEEE Security Privacy, vol. 1, no. 2, pp. 3342, Mar./Apr. 2003

[2] A. K. Jain, K. Nandakumar, and A. Nagar, "Biometric template security," EURASIP J. Adv. Signal Process., vol. 2008, pp. 113-129, Jan. 2008.

[3] SanjanaPrasad, P.Mahalakshmi, A.John Clement Sunder R.Swathi”Smart Surveillance Monitoring System Using Raspberry PI and PIR Sensor" International Journal of Computer Science and Information Technologies(IJCSIT)ISSN 0975-9646 Vol.5 (6),2014, $7107-7109$

[4] Anoop Mishra "Embedded Image Capturing \& Digital Converting Process using Raspberry pi System interfacing and Comparison of Generation 2 verses Generation 1 models in Raspberry pi” et al, /(IJCSIT) International Journal of Computer Science and Information Technologies, Vol. 6 (2), 2015, 1798-1801

[5] "Face Recognition based on Elastic Template," Beijing University of Technology, China, M H Yang, D J Kriegman, and N Ahuja. Detecting facesin images: a survey. IEEE Trans. on PAMI, 2002

[6] Dalal N. and Triggs B. 2005. Histograms of Oriented Gradients for Human Detection. In Proceedings of IEEE International Conference on Computer Vision and Pattern Recognition

[7] ] Liu Y., Yao J., Xie R., and Zhu S. 2013. Pedestrian Detection from Still Images Based on Multi-Feature Covariances. In Proceeding of the IEEE International Conference on Information and Automation

[8] Hussain S. and Triggs B. 2010. Feature sets and dimensionality reduction for visual object detection. In British Machine Vision Conference

[9] ] Jun B., Choi I., and Kim D, "Local Transform Features and Hybridization for Accurate Face and Human Detection", IEEE Transactions on Pattern Analysisand Machine Intelligence, 2013.

[10] R. Chellappa, C.L. Wilson, and S. Sirohey, "Human and machine recognition of faces: Asurvey," Proc. IEEE, vol. 83, pp. 705-740, 1995. 